\title{
ANALISE DA REVISTA DA ESCOLA DE ENFERMAGEM
} DA USP: PERIODO DE 1982 a 1986

\author{
Silvio Augusto Margarido * \\ Maria Sumie Koizumi** \\ Marli Alves Rolim*** \\ Ilza Marlene Kuae Fukuda***
}

MARGARIDO, S.A.; KOIZUMI, M.S.; ROLIM, M.A.; FUKUDA, I.M.K. Análise da Revista da Escola de Enfermagem da USP: período de 1982 a 1986. Rev. Esc. Enf. USP, São Paulo, 21(3):285-291, dez. 1987.

Em comemoração ao vigésimo aniversário da Revista da Escola de Enfermagem da USP, os autores, integrantes do Conselho Editorial da referida publicação, decidiram dar continuidade (anos de 1981-1986) ao trabalho anteriormente desenvolvido por STEFANELLI et alii's que teve como objetivo fazer uma análise retrospectiva de toda matéria publicada (1967-1981).

UNITERMOS: Periódico cientifico. Periódicos - Avaliação. Revista da Escola de Enfermagem da USP - História.

\section{INTRODUÇÃO}

Por ocasião do 20" aniversário da Revista da Escola de Enfermagem da USP (REEUSP), emergiu a necessidade de se fazer a análise retrospectiva da matéria publicada nesse periódico dada a importância da revista no contexto da bibliografia brasileira de Enfermagem.

Como se teve conhecimento de que um grupo de doutorandas da Escola de Enfermagem da USP (EEUSP) já havia realizado estudo semelhante até 1981, o grupo do Conselho Editorial propôs-se então completar essa análise, abrangendo o periodo de 1982 a 1986, utilizando a mesma metodologia.

No Editorial do primeiro número do volume deste ano de $1987^{2}$, "Vinte anos de vida" - a Sra. Maria Rosa de Sousa Pinheiro, ex-Diretora da Escola, encarregou-se de relatar as finalidades e as caracteristicas da Revista. Vale ressaltar que no início seu conteúdo foi mais voltado para a assistência e ensino, a partir de 1973, para a investigação científica.

- Enfermeiro. Auxiliar de Ensino do Departamento de Enfermagem em Saúde Coletiva da Escola de Enfermagem da USP - disciplina Finformagem em Doencas Transmissiveis.

* Enfermeira. Professor Assistente Doutor do Departamento de Enfermagem Méáico-Cirúrgica da USP - disciplina Infermagem Médico-Cirurgica.

*** Enfermeira. Prolessor Assistente do Departamento de Enfermagem Materno-Infantil e Psiquítrica da Escola de Enfermagem da USP - disciplina Enfermagem Psiquiátrica. 
Aspectos relevantes: $1^{\circ}-$ nunca houve interrupção na periodicidade das publicações e; $2^{\circ}$ - atualmente esta Revista conta com 7 assinantes de Portugal além dos assinantes no Brasil e com o esquema de permuta em vários países como: Estados Unidos (13), França (1), Suiça (1), Havaí (1), Portugal (1), India (2), Inglaterra (2), Japão (1), Itália (2), Filipinas (1), Espanha (2), Canadá (1), Alemanha (1), Zambia (1), Chile (1).

Em 1981, AGUIARI \& $\mathrm{HONDO}^{1}$, com o objetivo de identificar os títulos de periódicos mais consultados pelos usuários da biblioteca da EEUSP, perceberam que a REEUSP era o segundo periódico mais consultado naquela biblioteca, com $707(12,18 \%)$ consultas no período de janeiro/dezembro daquele ano.

Tais fatos mostram a importância da análise do conteúdo dessa publicação bem como as tendências da Enfermagem que nela foram retratadas ao longo dos anos.

\section{METODOLOGIA}

A metodologia utilizada foi a proposta, por TSUNECHIRO et alii ${ }^{4}$ e aplicada por STEFANELLI et alii ${ }^{3}$ para análise dos 15 primeiros volumes da REEUSP, correspondentes aos primeiros 15 anos de vida do periódico em questão.

Os itens referentes à estrutura desta Revista, no periodo de 1967 a 1981, foram analisados por STEFANELLI et alii ${ }^{3}$ e não sofreram alterações na sua estrutura nos anos subseqüentes. Desta forma, a análise do periodo 1982 a 1987 restringiu-se ao seu conteúdo.

\section{RESULTADOS E COMENTARIOS}

A matéria publicada no periodo analisado compreende 152 itens, o que mostra ter havido aumento proporcional em relação aos 15 anos anteriores, quando foi de 329 matérias (Tabela 1). Esta diferença deveu-se principalmente ao aumento de pesquisas realizadas com a finalidade de obtenção de título acadêmico e da publicação de algumas pesquisas, bem como, resumos de teses e de monografias e notas prévias.

Na Tabela 1 observa-se, ainda, que o número de artigos e pesquisas publicados é equivalente, representando, respectivamente, $34,87 \%$ e $32,89 \%$ de toda a matéria publicada. Embora não tenha ocorrido nenhuma alteração na linha editorial da Revista, é clara a tendência dos autores em produzir pesquisas, como, aliás havia sido previsto quando do lançamento da Revista.

Considerando que os artigos e as pesquisas representam $67,76 \%$ de toda a matéria publicada no periodo, pode-se dizer que eles caracterizam o conteúdo da Revista. Portanto, optou-se por analisá-los mais detalhadamente. 
TAB EL A 1

CONTEUDO DA REVISTA DA ESCOLA DE ENFERMAGEM DA USP (REEUSP), NO PERIODO 'DE 1982 A 1986.

\begin{tabular}{|c|c|c|}
\hline Conterido & $\mathbf{N}$ & $\%$ \\
\hline Artigo & 53 & 34.87 \\
\hline Pesquisa & 50 & 32,89 \\
\hline \multicolumn{3}{|l|}{ Outros } \\
\hline - editorial & 15 & 9,87 \\
\hline - nota prévia & 12 & 7,89 \\
\hline 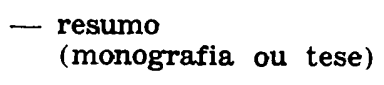 & 10 & 6,58 \\
\hline - palestra & 6 & 3,95 \\
\hline - discurso & 5 & 3,29 \\
\hline — informação & 1 & 0,66 \\
\hline Total & 152 & 100,00 \\
\hline
\end{tabular}

T A B E L A 2

DISTRIBUIÇAO DOS ARTIGOS E PESQUISAS SEGUNDO AREA DE ATUAÇAO E GRAU ACADEMICO DO AUTOR, PERIODO DE 1982 A 1986.

\begin{tabular}{|c|c|c|c|c|c|c|}
\hline \multirow{2}{*}{$\begin{array}{c}\text { Grau } \\
\text { Acadêmico }\end{array}$} & \multicolumn{4}{|c|}{ Area de Atuagáo } & \multicolumn{2}{|c|}{ Total } \\
\hline & Docência & Assistência & Ambos & Outro* & $\mathbf{N}^{9}$ & $\%$ \\
\hline Graduação & 18 & 2 & 1 & 8 & 29 & 28,16 \\
\hline Mestrado & 47 & - & - & - & 47 & 45,67 \\
\hline Doutorado & 16 & - & - & 一 & 16 & 15,53 \\
\hline Prof. Adjunto & 2 & - & - & - & 2 & 1,94 \\
\hline Prof. Titular & 4 & - & - & - & 4 & 3,88 \\
\hline $\begin{array}{l}\text { Sem especifi- } \\
\text { cação }\end{array}$ & 5 & - & - & - & 5 & 4,86 \\
\hline Total & $92(89$ & $2(1,94$ & $1(0$ & $8(7$, & 103 & $(100,00)$ \\
\hline
\end{tabular}

* Aluno de graduação ou pós-graduação.

Analisando-se a Tabela 2, verifica-se que a maior produção é resultante de trabalho de autores que atuam na área da docência $(89,32 \%)$ e com predomínio dos portadores de título de Mestre $(45,63 \%)$. 
Esta tendência da maioria dos autores estarem atuando na área de ensino também foi observado por STEFANELLI et alii ${ }^{3}$.

O grau acadêmico dos autores passou a ser sistematicamente divulgado neste período de 5 anos. $E$ possivel que isto tenha ocorrido por orientação do Conselho Editorial da Revista que julgara importante divulgar a titulação acadêmica obtida pelos autores que, na sua maioria, era constituída de enfermeiros.

Em segundo lugar aparecem os autores que não cursaram pós-graduação $(28,16 \%)$ e a seguir, aqueles com título de doutor $(15,53 \%)$, ambos atuando na área de ensino.

Neste periodo não houve alteração em relação ao número de trabalhos de autoria de doutores, comparado a do periodo anteriormente analisado ${ }^{3}$.

Além disso, verifica-se que das 50 pesquisas publicadas, $9(18,00 \%)$ explicitaram que o trabalho havia sido realizado com a finalidade de obtenção de título acadêmico.

Quanto aos autores de artigos e pesquisas, verifica-se que naturalmente o maior contingente continua sendo de docentes da EEUSP $(85,87 \%)$, tendência esta já verificada nos anos anteriores ${ }^{3}$. Os docentes da Escola de Enfermagem da USP, de Ribeirão Preto (EERPUSP) contribuiram com $8,69 \%$ da autoria dos artigos e pesquisas publicadas neste periodo, seguido de autores vinculados a outras instituições $(16,30 \%)$.

Pela Tabela 3 verifica-se que o enfoque dos artigos e pesquisas continua sendo princaplmente o hospitalar já encontrado por STEFANELLI et alii ${ }^{3}$. Porém, é interessante notar que na matéria de enfoque hospitalar, houve aumento no número de pesquisas em relação ao de artigos e que, nos de enfoque comunitário, o número de artigos superou o de pesquisas. Esta tendência foi contrária à encontrada nos anos anteriores ${ }^{3}$.

TABELA 3

DISTRIBUTÇAO DOS ARTIGOS E DAS PESQUISAS, SEGUNDO O ENFOQUE NO PERIODO DE 1982 A 1986.

\begin{tabular}{|c|c|c|c|c|c|c|}
\hline \multirow{3}{*}{ Enfoque } & \multicolumn{4}{|c|}{$P u b l i c a c a ́ o$} & \multirow{2}{*}{\multicolumn{2}{|c|}{ Total }} \\
\hline & \multicolumn{2}{|c|}{ Artigo } & \multicolumn{2}{|c|}{ Pesquisa } & & \\
\hline & No & $\%$ & $N^{9}$ & $\%$ & $\mathbf{N}^{e}$ & $\%$ \\
\hline Hospitalar & 21 & $(39,62)$ & 30 & $(60,00)$ & 51 & $(49,51)$ \\
\hline Comunitário & 13 & $(24,53)$ & 7 & $(14,00)$ & 20 & $(19,42)$ \\
\hline Ambos & 2 & $(3,77)$ & 2 & $(4,00)$ & 4 & $(3,88)$ \\
\hline IJenhum & 17 & $(32,08)$ & 11 & $(22,00)$ & 28 & $(27,19)$ \\
\hline Total & 53 & $(100,00)$ & 50 & $(100,00)$ & 103 & $(100,00)$ \\
\hline
\end{tabular}


Quanto à distribuição dos artigos e das pesquisas segundo as áreas (Tabela 4), percebe-se que as áreas mais abordadas continuam sendo a assistencial e a de ensino, como verificado anteriormente por STEFANELLI et alii ${ }^{3}$. Contudo, verifica-se que houve diminuição proporcional nas matérias da área asistencial, tanto nos artigos como nas pesquisas; por outro lado, houve aumento proporcional das pesquisas nas áreas de ensino e de administração.

TABELA 4

DISTRIBUIÇÃO DOS ARTIGOS E PESQUISAS SEGUNDO AREAS, NO PERIODO DE 1982 A 1986.

\begin{tabular}{|c|c|c|c|c|c|c|}
\hline \multirow[t]{2}{*}{ Area } & \multicolumn{2}{|c|}{ Artigo } & \multicolumn{2}{|c|}{ Pesquisa } & \multicolumn{2}{|c|}{ Total } \\
\hline & $\mathbf{N}$ & $\%$ & $\mathbf{N}$ & $\%$ & $\mathbf{N}^{9}$ & $\%$ \\
\hline Assistência & 25 & $(47,17)$ & 18 & $(36,00)$ & 43 & $(41,75)$ \\
\hline Ensino & 14 & $(26,42)$ & 12 & $(24,00)$ & 26 & $(25,24)$ \\
\hline Administração & 4 & $(7,55)$ & 8 & $(16,00)$ & 12 & $(11,65)$ \\
\hline Profissão & 5 & $(9,44)$ & 2 & $(4,00)$ & 7 & $(6,80)$ \\
\hline $\begin{array}{l}\text { Metodologia } \\
\text { de Pesquisa }\end{array}$ & 1 & $(1,87)$ & 4 & $(8,00)$ & 5 & $(4,85)$ \\
\hline Outras & 4 & $(7,55)$ & 6 & $(12,00)$ & 10 & $(9,71)$ \\
\hline Total & 53 & $(100,00)$ & 50 & $(100,00)$ & 103 & $(100,00)$ \\
\hline
\end{tabular}

A Tabela 5 mostra que na área assistencial não houve alteração quanto à distribuição nos vários sub-itens que caracterizam essa linha, se comparada a dos anos anteriores ${ }^{3}$.

$\mathrm{Na}$ linha de ensino, Tabela 5, os trabalhos sobre a avaliação do processo ensino/aprendizagem e as estratégias de ensino predominaram, mostrando uma evolução diferente da apresentada nos anos anteriores ${ }^{3}$.

Em relação à área de administração (Tabela 5), percebe-se que os trabalhos continuam voltados para as funções do enfermeiro e para os recursos humanos e materiais, porém mais dirigidos para a pesquisa.

$\mathrm{Na}$ área da profissão não se percebem linhas nitidas de evolução.

$\mathrm{Na}$ área metodologia de pesquisa foram identificadas 4 pesquisas sobre fundamentação teórica e 1 artigo sobre instrumentos e técnicas.

Como "outras áreas", somando 4 artigos e 6 pesquisas, foram incluídas as referentes ao currículo e experimentação com animais de laboratório. 
DISTRIBUIÇAO DOS ARTIGOS E DIAS PESQUISAS DAS AREAS ASSISTENCIAL, ENSINO, ADMINISTRAÇAO E PROFISSAO, SEGUNDO AS LINHAS, NO PERIODO DE 1982 A 1986.

\section{Linha}

Artigo
Pesquisa

Tota]

\section{ASSISTENCIAL}

- Planejamento da assisténcia

- Fundamentação para a assistência

- Necessidades ou problemas do paciente

- Educação para a saúde

- Sistematização da assistência

S u b - Tot a l

$\begin{array}{rrr}5 & 4 & 9 \\ 13 & 10 & 23 \\ 1 & 1 & 2 \\ 1 & - & 1 \\ 5 & 3 & 8 \\ 25 & 18 & 43\end{array}$

ENSINO

- Currículo

$\begin{array}{r}2 \\ 5 \\ 1 \\ 5 \\ 1 \\ \hline 14\end{array}$

- Avaliação do processo ensino-aprendizagem

- Método e té cnicas de ensino

- Estratégias de ensino

- Caracterização do aluno

- Recursos humanos

S u b - Tot a l

ADMINISTRAÇAO

- Funções do enfermeiro

- Recursos humanos e materiais

- Educação continuada

- Educação em serviço

S u b - Total

2

6

\section{PROFISSAO}

- Teorias, princípios e conceitos

- Enfermagem como prática social

- Filosofia e valores profissionais

S u b-Total

$$
\text { TOTAL }
$$

1
1
1
4

$\begin{array}{rr}5 & 6 \\ 3 & 4 \\ - & 1 \\ - & 1 \\ 8 & 12\end{array}$

$\begin{array}{rrr}3 & - & 3 \\ - & 1 & 1 \\ 5 & 1 & 3 \\ 48 & 2 & 7 \\ & 40 & 88\end{array}$

\section{CONCLUSOEES}

A análise comparativa do conteúdo da REEUSP, no periodo de 1982 a 1986, em relação aos 15 anos anteriores, mostrou que:

- houve aumento proporcional de pesquisas publicadas, embora não tenha havido alterações na linha editorial; 
- a produção científica continua centrada nos autores com título de mestre que atuam na área de ensino: continua sendo a maior, o que não é de se estranhar, uma vez que o número de cursos de Mestrado ainda é maior que o de Doutorado;

- o enfoque dos artigos e pesquisas continua a ser o hospitalar;

- as áreas de predileção dos autores continuam sendo a assistencial e a de ensino;

- houve aumento proporcional no número de pesquisas das áreas de ensino e administração.

MARGARIDO, S.A.; KOIZUMI, M.S.; ROLIM, M.A.; FUKUDA, I.M.K. Contents of Revista da Escola de Enfermagem da USP: period of 1982 to 1986. Rev. Esc. Enf. USP, São Paulo, 21(3):285-291, Dec. 1987.

To celebrate the 20th aniversary of the Revista da Escola de Enfermagem da USP, the authors decided to extend to the years of 1982-1986 the analysis of the first fifteen years of the periodical, above published, made by STEFANELLI et alii.

UNITERMS: Scientific periodicals. Periodicals - Evaluation. Revista da Escola de Enfermagem da USP - History.

\section{REFERENCIAS BIBLIOGRAFICAS}

1. AGULARI, C.S.A.L. \& HONDO, M.M.N. A utilização dos periódicos na Biblioteca da Escola de Enfermagem da USP. Rev. Eso. Enf. USP, São Paulo, 16(1):5-16, abr. 1982.

2. PINHEIRO, M.R.S. Editorial: «Vinte anos de vida». Rov. Esc. Enf. USP, São Paulo, 21 (1) :1-2, abr. 1987.

3. Stefanell, M.C.; elsas, B.X.; CarValho, D.V.; tSunechiro, M.A.; POSSO, M.B.S.; LUI, M. do C. Análise da Revista da Escola de Enfermagem da USP: periodo de 1967 a 1981. Rov. Esc. Ent. USP, São Paulo, 21(3), dez. 1987. (no prelo).

4. TSUNECHIRO, M.A.; CARVALHO, D.V.; POSSO, M.B.S.; ELSAS, B.X.; LUI, M. do C.; STEFANELLI, M.C. Instrumento para analise de periódico de enfermagem. Rev. Fisc. Enf. USP, São Paulo, 17(2):107-17, ago. 1983.

Recebido para publicaçăo em novembro de 1987.

Aprovado para publicação em dezembro de 1987. 